\title{
NOTAS DE EPIGRAFÍA HISPÁNICA
}

\author{
POR \\ JUAN MANUEL ABASCAL PALAZÓN \\ Universidad de Alieante
}

\section{RESUMEN}

Este breve articulo contiene correcciones y adiciones a un conjunto de inscripciones latinas de Hispania (provincias de Alicante, Badajoz, Burgos, Cáceres, Cuenca, Guadalajara, Orense. Valencia y territorium de Aquae Flauiae). Salvo un grafito, aủn inédito, y un texto prácticamente desconocido de Sagunto, las observaciones que se hacen se refieren a lecturas o bibliografia de textos ya publicados.

\section{SUMMARY}

This brief paper contains corrections and addenda to a collection of Latin inscriptions of Hispania (provinces of Alicante, Badajoz, Burgos, Cáceres, Cuenca, Giuadalajara, Orense, Valencia and territorium of Aquae Flauiae). Except for one graffito, not yet published, and another practically unknown text from Sagunto, all the observations that we make refer to readings and bibliography of already published texts.

Las páginas que siguen son notas de lectura sobre inscripciones ya conocidas si se exceptúan dos textos, de Alicante y Valencia respectivamente, que no han sido publicados hasta la fecha. En la mayor parte de los casos nuestras observaciones se dirigen a precisiones onomásticas e identificacion de textos repetidos, pero también incluyen especialmente en los referentes al territorium de Aquae Flaviae, las consideraciones de tipo bibliográfico. El conjunto de inscripciones aqui reseñadas corresponde mayoritariamente a la provincia Tarraconense, aunque no faltan algunos ejemplos lusitanos.

\section{PROVINCIA DE ALICANTE}

n 1. Alicante, «Tossal de Manises». Inédito.

En la Guía del Museo de Alicante de 1959 (1), J. Lafuente citó dos pondera de barro cocido inéditos, procedentes de las excavaciones del municipium asentado en el Tossal de Manises, Lucentum en Plinio, que presentaban inscripción en su parte superior. El primero de ellos ya ha sido dado a conocer (2), mientras el segundo ha aparecido en una reciente revisión de los fondos del Museo (3).

El nuevo ejemplar tiene unas dimensiones máximas de $8,3 \times 6,1 \times 4,1 \mathrm{~cm}$ y un peso de 320 gramos; en su cara superior ostenta una breve inscripción incisa en una sola línea cuyas letras miden $1,3 \mathrm{~cm}$ de altura. Se conserva en el Museo Arqueológico Provincial de Alicante (Inv. TM-1287) y el texto dice:

Lucan[i]

1. J. Lafuente, Museo Arqueológico Provincial de Alicante. Catálogo Guia, Alicante 1959. $51, n^{\circ} 134$

2. Museo de Alicante, Inv. TM-1286 $(280,01$ gramos). M.A. Rabanal y J.M. Abascal, Inscripciones romanas de la provincia de Alicante, Lucentum $4,1985,198, n^{\circ} 13$, fig. $8(=\mathrm{AE}$ 1986, 437 y HEp. 1, 67) = L. Abad y J.M. Abascal, Textos para la Historia Antigua de Alicante. Edad Antigua, Alicante 1992, 164 s., $n^{\circ}$ ID 15.

3. Agradecemos a D. José Antonio López Mira la noticia sobre la existencia de la pieza asi como su descripción y los datos que aquí ofrecemos.

$\mathrm{n}^{\circ} 2$. Alicante. Inscripción procedente de Italica.

CIL II 1126, con la bibliografia anterior; A. Engel, Revue Archéologique, ser. III, vol. 29, 1896, 204, n. 3 =EE 9,354a (= M.A. Rabanal y J.M. Abas- 
eal, Dos nuevas inscripciones y algunas correcciones a la epigrafia romana de la provincia de Alicante, Lucentum 5, $1986,170 \mathrm{s..} \mathrm{n}^{\circ} 3+\mathrm{L}$. Abad y J.M. Abascal, Textes para la Historia Antigua de Alicante. Eclad Antigua. Alicante 1992, 114, n"57).

El 13 de mar/o de 1894 , A. Engel vio esta inscripcion en la colección alicantina del Marqués de Bosch, de donde pasó a Ephemeris Epigraphica. en donde se supuso un nuevo texto encontrado prope Alicante; con tan escasa información fue incluida en los repertorios locales, pero hoy no hay duda de que se trata del texto italicense CIL II 1126 , del que Hübner indica que fue trasladado al domicilio alicantino del Marqués de Casa Rojas; el título de Conde de Casa Rojas (no marquesado, como indica Hübner) fue establecido por Carlos IV en 1790, recayendo finalmente en el Marquesado de Bosch de Arés, lo que explicaría la aparente divergencia entre las noticias de Hübner y Engel.

\section{PROVINCIA DE BADAJOZ}

\section{$n^{\circ}$ 3. Mérida}

A. Garcia y Bellido, Parerga de arqueologia y epigrafia hispano-romanas, $A E A 33,1960$, $167-193$, p. 182, n० 7, fig. 33 (= HAE 1842).

De este fragmento de lápida en mármol, hallado en la Alcazaba, se conserva únicamente el ángulo superior derecho con el final de la primera línea y parte de la segunda. El texto visible en el dibujo que publicó A. Garcia y Bellido dice:

$$
\begin{aligned}
& {[-]+\text {. SPEN }} \\
& {[-]++}
\end{aligned}
$$

Habida cuenta de que delante de la primera letra visible se conserva una interpunción, y que por ser la primera línea de texto debe contener una fórmula ono mástica, proponemos leer entre 1.1 y $1.2 \mathrm{el}$ nombre Spen [dusa] (1). Esta restitución puede ponerse en relación con un texto segobricense (2) recientemente revisado por nosotros en el que creimos identificar Spendusa como un hapax en la epigrafia hispánica (3), suposición que debe rechazarse a la vista de este texto.

1. H. Solin, Die griechischen Personennamen in Rom. Ein Namenbuch, Berlin 1982, $1267 \mathrm{y}$ 1338.

2. J. de Dios de la Rada y F. Fita, Excursión epigráfica a las ruinas de Cabeza de Griego, $B R A H$ $15,1889,127=$ CIL II 5876 (=ILER 907); P.
Quintero. Licles. Excasaciones efectuadas en distintas épocas y noticia de algunas antigüc-

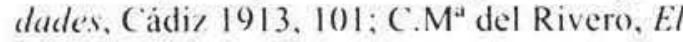
lapidario del Mhuseos Arquesológicon Nacional. Madrid 1933, 15. n" 44; J.M* Blázque\%. Religiones primitivas de Hispania I. Fuentes literarias y epigraficas, Roma 1962.70, fig. 10: M. Almagro. Segohriga II. Inscripciones ihèrcas, latinas paganas y latinas cristianas. Excas: Arq. en España 127, Madrid 1984, n"16.

3. J. M. Abascal, Una officina lapidaria en Segobriga. El taller de las series de arcos, $H A n t$ 16, 1992, 309-343, p. 311, n” 1, fig. 1.1; id., Pendusa, un falso teónimo de la Celtiberia meridional, Hom. Francisco Presedo, Sevilla 1993 (en prensa), con foto.

$n^{\circ} 4$. Mérida.

E. Garcia Sandoval, Informe sobre las casas romanas de Mérida y excavaciones en la "Casa del Anfiteatrom, Excav: Arq. en España 49, Madrid 1966. 13, lám. XVI (= HAE 2580): M. Almagro Basch, Guia de Mérida (9 $9^{a}$ ed.), Valencia 1981, 96, lám. LXI (= HAE 2682); A. Blanco, Mosaicos romanos de Mérida. Corpus de mosaicos romanos de España, vol. I, Madrid 1978, 34, lám. 26.

Se trata de un breve texto epigráfico en el ángulo superior izquierdo de un mosaico con una escena central de Baco y Ariadna. Lacónicamente dice:

\section{EX OFFICINA \\ ANNIPONI}

Tradicionalmente el nombre del artista se ha leido como una expresión en genitivo de nomen y cognomen, pero ni la disposición del texto aconseja tal distinción ni los paralelos onomásticos la permiten. Disponemos hoy de una referencia africana que ha tenido entrada en el reciente repertorio onomástico de H., Solin y O. Salomies (1), con los nombres de dos obispos fechados hacia el año 400 d.C., cuya nombre es Annibonius. Con este paralelo hay que descartar que el autor del mosaico emeritense sea un Annius y suponer que Anniponius y Annibonius son formas sorda y sonora de un mismo cognomen.

1. H. Solin y O. Salomies, Repertorium nominum gentilium et cognominum Latinorum, Hildesheim 1988, 293.

\section{$n^{\circ} 5$. Mérida.}

CIL II $584=3049 ;$ F. Fita, BRAH 36, 1900, 445

+ C. Fernández Duro, ibid., 523 s. (= EE 9, p. 26 y 120 . 
La estela sepulcral de Pompeia Rodope hallada en Merida fue trasladada a Guadalajara, en donde llegó a estar empotrada en casa de D. Casimiro Mogilnicki (Calle Baja de Barrionuevo, 56, actualmente ( / Ingeniero Mariño). Con esta información se publicaron sendas noticias en el Boletin de la Real Academia de la Historia, y a partir de ellas Hübner identificó la pieza como emeritense.

Con el ánimo de confirmar la existencia o desaparición del monumento, hemos indagado sin éxito en la localización citada, con lo que la pieza se puede dar por desaparecida (1).

1. Agradecemos las gestiones realizadas para la localización del callejero antiguo al Dr. Sánchez-Lafuente (Universidad de León).

\section{PROVINCIA DE BURGOS}

$n^{\circ} 6$ Poza de la Sal.

J. Martínez Santa-Olalla, Anuario de Prehistoria Madrileña 2-3, 1931-32, 136 s. y 155, lám. VII, fig. $3^{\mathrm{a}}$; M. Martínez Burgos, Catálogo del Museo Arqueológico Provincial de Burgos, Madrid 1955 , $66, \mathrm{n}^{\circ} 114$; J.A. Abásolo y $\mathrm{M}^{\mathrm{a}} \mathrm{L}$. Albertos, Acerca de unas inscripciones de Poza de la Sal, BSEAA 1976, 393-407, p. 403 s., lám. 111.2 (= AE 1976, 321); P. de Palol y J. Vilella, Clunia II: La epigrafia de Clunia, Excav.Arq.Esp. 150, Madrid 1987, 35, Add. I (= AE 1988, 773).

La inscripción no es inédita como sugieren Palol y Vilella. Su lugar de procedencia no es la ciudad de Clunia, sino el lugar conocido como «La Vieja», en Poza de la Sal (Burgos), que exploró J. Martinez Santa-Olalla; a él se debe la información sobre el hallazgo del monumento poco antes de 1931.

$n^{\circ} 7$. Salas de los Infantes.

S. González de Madrid y F. Wattemberg, Museo Arqueológico Provincial. Guia del visitante, Valladolid 1960, 7; J.A. Abásolo, Epigrafia romana de la región de Lara de los Infantes, Burgos 1974, 149. $\mathrm{n}^{\circ}$ 206; E. Wattemberg, Museo Arqueológico de Valladolid, Valladolid 1990, 22. Museo de Valladolid, sala 7.

El nombre del dedicante de esta inscripción ha sido leido tradicionalmente como Valerius Prissus Valeriani f(ilius); sin embargo, hay motivos para suponer que su cognomen no es Prissus sino Pressus: en efecto, al comienzo de la segunda línea hay una breve fractura en la que debía figurar la vocal en cuestión; en la parte inferior de esa fractura se observa un breve trazo horizontal que no corresponde al tipo de I que presenta el texto, y que conviene a una $\mathrm{E}$ mu estilizada y de trazos horizontales cortos como las que figuran en otras líneas del mismo.

Esta modificación permite dejar de considerar el cognomen Prissus como un hapax no sólo hispánico sino latino; al mismo tiempo, Pressus es un cognomen latino ( 1 ) bien conocido en la Peninsula lbérica (2).

1. I. Kajanto, The Latin cognomina, Roma 1982 (rptd.), 354, que recoge dos testimonios tarraconenses de CIL II ( 5812 y 5838 ); H. Solin y O. Salomies, Repertorium nominum gentilium et cognominum Latinorum, Hildesheim $1988,383$.

2. CIL II 5812: Severius Pressus (Sasamón, Burgos); CIL II 5838: Mummius Pressus (Puebla de (astro, Huesca): CIL II $2676+$ p.912: [A]emilius [P]ressus (León); J.A. Abásolo, Epigrafia romana de la región de Lara de los Infantes, Burgos 1974, 68, $\mathrm{n}^{\circ} 72$ : Visadus Pressus Elaesi Pulliani f(ilius) (Lara de los Infantes, Burgos).

$n^{\circ} 8$. Coruña del Conde.

AE 1988,804 recensiona la inscripción publicada por P. de Palol y J. Vilella, Clunia II: La epigrafia de Clunia, Excav.Arq.Esp. 150, Madrid 1987, $78, n^{\circ} 96$, pero aunque ofrece correctamente los datos de localización y medidas de la pieza, trasmite el texto de la inscripción $n^{\circ} 98$ del mismo catálogo.

\section{PROVINCIA DE CÁCERES}

n० 9. Coria.

R. Thouvenot, Note sur trois inscriptions lusitaniennes, REA $42,1940,529-531$, p. 530 (= AE 1941, 132); S.G. Armistead, Tres nuevas lápidas hispano-romanas de Coria, $A E A$ 58, 1985, 155-158, $\mathrm{n}^{\circ} 3$, p. 156 s. (= AE 1985,538$)$.

Pese a las apreciables diferencias de lectura entre ambos editores, se trata de dos versiones de la misma inscripción:

$\begin{array}{ll}\text { Thouvenot } & \text { Armistead } \\ \text { PINEANVS } & \text { PINTAMVS } \\ \text { FVRnACI } & \text { TVRRACIAE } \\ \text { AN XL BO } & \text { AN XLV BO } \\ \text { ETE LAVRI } & \text { ETEIA VRI } \\ \text { EN AN LX H } & \text { ENI AN LX H } \\ \text { S S S V T L } & \text { S S S V T L }\end{array}$


Como puede observarse por la comparación de ambas lecturas, la diferente observación de trazos horizontales y la interpretación de rasgos dudosos llevaron a S.G. Armistead a suponer inédita la inscripción.

\section{PROVINCIA DE CUENCA}

n"10. Carboneras.

A. Rodriguez Colmenero, Cuenca romana. Contribución al estudio epigráfico, Lucentum 1, 1982. $203-253, n^{\circ} 50$, p. 222 ( $=$ AE 1982,609$)$.

La transcripción correcta de 1.2 debe ser Peren$n i$, tal y como figura en el monumento, sin necesidad de desarrollar Perenniu(s), como quiere el primer editor.

\section{n'11. Valeria.}

CIL II 3198, con la bibliografia anterior ( $=$ F. Fita, Inscripciones romanas y griegas de Cartagena, Almazarròn. Pego, Valera, Herramelluri, Córdoba, Vélez Rubio y Vera, BRAH 52, 1908,505 - 530, p. 516, n' 26 y 518; ILER 2480): T. Muñoz y Soliva, Memoria de todos los señores obispos que han regido la diócesis de Cuenca, Cuenca 1860 , 439; M. López. Memorias de Cuenca y su obispado, Cuenca 1953 (edición de A. González Palencia), 134, n'22 $=$ A. Rodriguez Colmenero, en M. Osuna et alii, Valeria romana, Cuenca 1978, 113 e $i d$., Cuenca romana. Contribución al estudio epigráfico, Lucentum 1, 1982, 203-253, p. 213, n² 27 , lám. II.22).

Esta inscripción fue publicada por Fita a comienzos de siglo con dos lecturas completamente distintas (op. cit.. pág. 516 y 518 ) indicando que estaba grabada en un vaso de alabastro. A. Rodriguez Colmenero la dio a conocer de nuevo a partir de un dibujo de M. López sin percibir la existencia de la edición previa de Fita ni el detalle de que el dibujo de López eliminaba las dudas sobre la existencia de ese hipotético vaso de alabastro reseñado por el ilustre jesuita.

\section{PROVINCIA DE GUADALAJARA}

$\mathrm{n}^{\circ}$ 12. Ciruelos del Pinar.

J.M. Abascal, Epigrafia romana de la provincia de Guadalajara, Wad-al-Hayara 10, 1983, 81 s., $\mathrm{n}^{\circ}$ 25 , fig. $1, n^{\circ} 25$ y fig. $16 \mathrm{~b}$.

Este fragmento de inscripción en pésimo estado de conservación presenta tan sólo restos de cuatro letras que en su momento identificamos como RENI: por la posición que ocupaban supusimos que se trataba de un inicio de palabra, pero no supimos determinar a cuál podria pertenecer.

Amistosamente, el Dr. L.A. Curchin (Univ. Waterloo. Ontario, Canadá) nos ha sugerido (1) interpretar estas pocas letras a la luz de varias inscripciones burgalesas y de algunos otros testimonios numnismáticos y epigráficos; efectivamente. parece probada la existencia de un nomen Renius no recogido por Schulze, pero que seria una variante del bien conocido Rennius, que aparece en Hispania sobre inscripciones de Lara de los Infantes (2). Miñón (3) y Revilla del Campo (4), todas ellas dentro de la provincia de Burgos. En apoyo de esta suposición invoca acertadamente Curchin la leyenda C. RENI con que aparece un magistrado monetal del año 138 a.C. (5).

La hipótesis de Curchin puede confirmarse si traemos a colación los testimonios epigráficos que en Hispania muestran la simplificación de estos grupos de consonantes geminadas. Nomina como Annius/a y Herennius/a aparecen escritos con una solo $\mathrm{N}$ en diversas ocasiones. Sin necesidad de alargar el argumento se pueden citar Anius Silv $(-$ ) en Rosinos de Vidriales (Zamora), Herenia C.f. Severa en Cáceres, Herenia Fecunda en Medellin (Badajoz), Ania Poudaum [-?] en Garray (Soria), el reciente testimonio de Anius Calistus en Munigua (AE 1989. 411), etc.

En consecuencia, y aceptando la opinión de Curchin, la inscripción de Ciruelos del Pinar podria considerarse como el epitafio de un Renius.

1. En carta de 31 de enero de 1991.

2. J.A. Abásolo, Epigrafia romana de la región de Lara de los Infantes, Burgos 1974, $\mathrm{n}^{\circ} 40$ : Rennius Pat(ernus).

3. J.A. Abásolo, BSAA 50, 1984, 197, $\mathrm{n}^{\circ} 6=\mathrm{AE}$ 1984,564: L(ucius) Rennius [-].

4. J.A. Abásolo, Epigrafia romana de la región de Lara de los Infantes, Burgos 1974, n²01: L. Rennius Renni f. y Rennius Valerianus.

5. M.H. Crawford, Roman Republican Coinage, Cambridge 1974, 264.

\section{PROVINCIA DE ORENSE}

(Vid. infra inscripciones de Aquae Flauiae)

Entre el conjunto de las inscripciones pertenecientes al territorium de Aquae Flauiae se encuentra un apreciable número de las orensanas. Inclui- 
das gran parte de ellas en el reciente estudio de $\mathrm{A}$. Rodriguez (olmenero (en adelante citado como AF: vid. infira), anotamos aqui las observaciones correspondientes a los monumentos de la provincia de Orense. La asignación topográfica se ha realizado a partir del libro de $\mathrm{M}^{\mathrm{a}}$ Pilar de Torres et alii, Las parroquias de Galicia. Cartografia y estadisticas, Santiago de Compostela 1985.

n'13. Concello de San Amaro, Eiras

AF, $182 \mathrm{~s}, \mathrm{n}^{\circ}$ 105.- La inscripción apareció reseñada en HAE 2721 y AE 1974, 408.

n"14. Concello de San Pedro de Montes, San Pedro de Montes

AF, 93, n" 51.- A la bibliografía citada, añadase CIL II 2611 (=ILER 213). Los comentarios a la lectura y la bibliografía están recogidos en A. Tranoy. Galice, 311 , npta 34 .

n" 15. Concello de Xinzo de Limia, Mosteiro de Ribeira

AF, 178, n०101 (= HEp 2,541).- La inscripción fue publicada por Fita, Tres lápidas romanas de Mosteiro de Riveira, $B R A H 58,1911,513-514$, que no aparece en la bibliografia final y se cita abreviadamente en pág. 178, con datos de Marcelo Macias; se conserva en el Museo de Orense. El l.l se ve claramente Peregrinu[s] y no Perecrinu(s) como sostiene Rodriguez Colmenero. Curiosamente, tras incluir este texto, procedente de Mosteiro, el autor omite CIL II 2566, que procede del mismo lugar, y no de Sta. Maria de Ribeira como alegaba Hübner.

$\mathrm{n}^{\circ}$ 16. Concello de Xinzo de Limia, Mosteiro de Ribeira

AF, 290, $n^{\circ} 179(=$ HEp 2, 542). Es casi con toda probabilidad la inscripción CIL 112565.

\section{PROVINCIA DE VALENCIA}

En 1925, Vicente Castañeda dio a conocer un nutrido grupo de cartas redactadas por Fray Luis Galiana y por algunos de sus corresponsales con diversos personajes de su tiempo (V. Castañeda, Cartas eruditas de Fray Luis Galiana, y de otros autores, recopiladas por él mismo, $B R A H 87,1925$, 612-668). La mayor parte de esa correspondencia se refería a temas eruditos ligados con la antigüedad de las tierras valencianas, y especialmente a los hallazgos que se producian regularmente en las ruinas de Murviedro (Sagunto, Valencia).

Dos de esas cartas tienen un especial interés epi- gráfico por no haber sido empleadas hasta la fecha en la elaboración de los estudios epigráficos sobre Saguntum, y por contener datos de cierta relevancia sobre el hallazgo y contenido de cinco textos fragmentarios.

La primera de estas epistolas, de 4 de enero de 1765 hace referencia al halla/go de nueve sepulcros de inhumación en 1759 baso el solar de la casa de la viuda de Francisco (asasús, en el arrabal de la Trinidad: la segunda carta, redactada el 7 de febrero de 1765, contiene precisiones sobre el hallazgo citado y sobre el de otras tres sepulturaso en la casa de D. Pedro Tovar, antigua calle Real, $n^{\circ} 25$ y posteriormente C/José Antonio, con el mismo número.

La información suministrada por estas cartas permite precisar el lugar de hallazgo de estos fragmentos, incluye uno probablemente inédito y descarta la identidad entre otros dos ya conocidos.

n* 17. Sagunto

CIL II 3925 + ELSagunto 136, con el resto de la bibliografia. Añádase ahora V. Castañeda, $B R A H 87$, $1925,613-616$ y 638-640.

Procede de los sepulcros hallados en 1759 en casa de la viuda de Francisco Casasús, en el arrabal de la Trinidad. Fue el único hallazgo epigráfico del conjunto y se trasladó a casa de D. Pedro Tovar (Calle Real, $n^{\circ} 25$ ), en donde quedó empotrada en la fachada. La carta que detalla el hallazgo fue enviada a Matias Perelló por el propio Luis Galiana el 4 de enero de 1765, y la ubicación definitiva del monumento se recoge en la misiva que Pedro Juan Miró envió a Gerónimo Espuig el 7 de febrero de 1765.

Ambos textos eliminan definitivamente la sospecha de $P$. Beltrán sobre la identidad de este texto con ELSagunto $n^{\circ} 259$, ya que de ambos se ofrecen noticias en estos documentos.

\section{$n^{\circ}$ 18. Sagunto}

CIL II 3919 (= ILER 6626) + ELSagunto 173, con el resto de la bibliografia. Añádase ahora $\mathrm{V}$. Castañeda, BRAH 87, 1925, 638-640.

Al hacer la casa de D. Pedro Tovar (Calle Real, $\mathrm{n}^{\circ} 25$ ) aparecieron tres sepulcros de inhumación formados por materiales epigráficos reaprovechados, a juzgar por la descripción que se da de los hallazgos. En el primero de ellos apareció el presente texto, según carta de Pedro Juan Miró a Gerónimo Espuig el 7 de febrero de 1765 .

\section{$\mathrm{n}^{\circ}$ 19. Sagunto}

El Sagunto 259, que la supone inédita. Añádase a la bibliografia V. Castañeda, $B R A H 87,1925,638$ 640 . 
Según la carta de Miro a Espuig de 7 de febrero de 1765 apareció en la segunda de las tumbas de la casa de Pedro Tovar (Calle Real, n" 25), formando el lateral de la misma. Véase lo dicho para la inscripción n" 10 sobre la imposibilidad de identificar este texto y El Sagunto 136.

n"20. Sagunto

(II. II $3830+$ ELSagunto 17, con el resto de la bibliografia. Añadase a ella $\mathrm{V}$. Castañeda, $B R \cdot A H$ 87, 1925, 638-640.

Según la carta de Miró a Espuig de 7 de febrero de 1765 , este monumento formaba uno de los extremos de la tercera sepultura de la citada casa de D. Pedro Tovar (Calle Real, $\mathrm{n}^{\circ} 25$ ).

$\mathrm{n}^{\circ}$ 21. Sagunto

V. Castañeda, BRAH 87, 1925, 638-640.

Este pequeño fragmento, que la carta de Miró a Espuig ( 7 de febrero de 1765) sitúa en el lateral de la tercera sepultura de la casa de Pedro Tovar (Calle Real, $n^{\circ} 25$ ), no ha tenido entrada hasta el presente en los repertorios epigráficos de la ciudad. La mencionada carta sólo indica que el texto los formaban «tres grandes iniciales» con las letras A.V.I. (la puntuación es original), sin que podamos precisar su contenido.

\section{LAS INSCRIPCIONES DE AQUAE FLAUIAE}

El conjunto epigráfico de Aquae Flauiae y su territorio posee una extraordinaria riqueza y ha sido objeto de un amplio estudio en fecha reciente por parte de A. Rodriguez Colmenero (Aquae Flaviae I. Fontes epigráficas, Chaves 1987, 2 vols.; en adelante AF); este trabajo fue objeto de una recensión por parte de J. M. Garcia en Conimbriga 27, 1988 , 211-216, que incidia especialmente en la lectura de algunos epigrafes. Por nuestra parte, señalamos a continuación algunas correcciones y adiciones de tipo bibliográfico sin ánimo de ser exhaustivos, sino citando únicamente trabajos especificos y precisando la procedencia de algunos textos.

\section{DISTRITO DE BRAGA}

Concelho de Braga

Braga

$\mathrm{n}^{\circ}$ 22. AF, 623 ss., $n^{\circ} 464$. Añádase a la bibliografia CIL II 2419 (= ILER 938; HAE 472) y EE 8,115.

\section{DISTRITO DE BRAC}

Concelho de Bragança

Castro de Avelas

$n^{\prime \prime}$ 23. AF, 333, n" 206. Esta inscripción (CIL II $2609+$ p. 909$)$ no procede de (haves, sino de Castro de Avelas (conc. y distrito de Bragança). (fi: A. Tranoy, Galice, 52, nota 116 .

Concelho de Miranda do Douro

Aldeia Nova

$n^{\circ}$ 24. AF, 282 ss., n' 175 . No es EE 8, 28 sino EE 8,128 y no 9.110 sino 9, p. 110 .

\section{DISTRITO DE SETUBAL}

Concelho de Alcacer da Sal

Alcacer da Sal

$n^{\circ} 25$. AF, 371 s., n² 240. Contra la opinión del autor, la inscripción procede de Alcácer da Sal. Se conserva en el Museo de Alcácer da Sal, donde la localizó Leite de Vasconcelhos en 1895 y pasó a editarse en EE 8, 4. Además de ésta referencia, añádase a la bibliografía J. D’Encarnaçao, Inscriçoes romanas do conventus Pacensis, Coimbra 1984, 262 ss, $\mathrm{n}^{\circ} 189$ con lo anterior y F. Bandeira, AP, n.s. 3, 1956, 87-107.

\section{DISTRITO DE VILA REAL}

Concelho de Chaves

Chaves

$n^{\circ}$ 26. AF, 55, $n^{\circ}$ 20. Añádase a la bibliografia $F$. Rusell, Viriatis 1, 1957, p. 100, n¹.

$\mathrm{n}^{\circ}$ 27. AF, $116 \mathrm{~s} ., \mathrm{n}^{\circ} 67$. Añádase a la bibliografia L. Fernández Fuster, La fórmula «ex visu» en la epigrafia hispánica, $A E A 80,1950,279-291=\mathrm{HAE}$ 34 con dibujo y AE 1933, 23.

$\mathrm{n}^{\circ}$ 28. AF, 222, $\mathrm{n}^{\circ}$ 129. Es CIL II 2471 (= ILER 676) con la bibliografía y comentarios en A. Tranoy, Galice, 303, nota 322 y 366, nota 18; véase además P. Le Roux y A. Tranoy, Conimbriga 23, 1984,35 , nota 50 .

$\mathrm{n}^{\circ}$ 29. AF, $295 \mathrm{~s}, \mathrm{n}^{\circ} 182$. No es ILER $4506 \mathrm{ni} 6129$ como se cita, ya que ambos corresponden a la ins- 
cripción n"181 del mismo catálogo, procedente de Orense. Añadase a la bibliografia A. Tranoy, Gali(e, 69 , nota 251 con cuya lectura coincide.

$n^{\circ}$ 30. AF, $368 \mathrm{~s} ., n^{\circ} 237$. Se trata de CIL. II 2488.

Concelho de Chaves

Eiras

$n^{\circ}$ 31. AF, 332, $n^{\circ}$ 204. Se trata de la inscripción CIL II 2481 (= ILER 6572).

Concelho de Chaves

Outeiro Seco

$\mathrm{n}^{\circ}$ 32. AF, 113, $\mathrm{n}^{\circ}$ 65.- No es CIL II 2472 sino 2473. Añádase a la bibliografía $\mathrm{AE} 1955,233$ y $\mathrm{AE}$ 1959,84 .

Concelho de Chaves

Redondela

$\mathrm{n}^{\circ}$ 33. $\mathrm{AF}, 298 \mathrm{~s} ., \mathrm{n}^{\circ}$ 183. Añadase a la bibliografia EE 9, p. 102.

Concelho de Chaves

Samaioes

$n^{\circ}$ 34. AF, 168 s., $n^{\circ} 95$ y AF, $238, n^{\circ} 140$. Se trata de dos versiones de la misma inscripción, en ningún caso inédita, pues fue publicada en CIL II 2475. Vid. además id., Veleia 2-3, 1985-6 = AE 1987 , $562 \mathrm{~b}$.
Concelho de Chaves

San Pedro Agostem

n" 35. AF, 222, $n^{\circ}$ 130. Se trata de CIL II 2470.

Concelho de Chaves

Tronco

$\mathrm{n}^{\circ}$ 36. AF, 46, n" 14. Se trata de la inscripción EE $9,274$.

$n^{\circ}$ 37. AF, 321, n¹98. Se trata de EE 9, 272.

$n^{\circ}$ 38. AF, 350 s., $n^{\circ} 221$. Se trata de EE 9, 273.

Concelho de Valpaços

Fiaes

$n^{\circ}$ 39. AF, 70, n 32. Es CIL II 2468 y 5615.

$n^{\circ}$ 40. AF, 369 s., $n^{\circ} 239$. Se trata de CIL II 2496 (= ILER 309)

Concelho de Valpaços

Frioes

$\mathrm{n}^{\circ}$ 41. AF, 70, $\mathrm{n}^{\circ}$ 33. No es CIL II 1495 sino 2495.

$\mathrm{n}^{\circ}$ 42. AF, 246, $\mathrm{n}^{\circ}$ 148. Es el texto de CIL II 2493. 